\title{
Viewpoint \\ Aspirin and breast cancer prevention - a link to ER status
}

Catherine R Tait

\author{
Molecular Medicine Unit, Clinical Sciences Building, St James's University Hospital, Beckett Street, Leeds, UK \\ Corresponding author: Catherine Tait, cathytait@doctors.org.uk \\ Published: 9 August 2004 \\ Breast Cancer Res 2004, 6:211-212 (DOI 10.1186/bcr923) \\ (C) 2004 BioMed Central Ltd
}

\begin{abstract}
Introduction
Speculation that nonsteroidal anti-inflammatory drugs (NSAIDS) may act to decrease the risk of breast cancer has been growing over recent years and prospective results from the Women's Health Initiative have provided further confirmation of this hypothesis [1]. This risk reduction has never previously been linked to oestrogen (ER) or progesterone (PR) receptor status, however, a recent population-based case control study [2] has demonstrated an inverse relationship between aspirin use and breast cancer in hormone receptor positive tumours.
\end{abstract}

\section{COX and prostaglandins}

NSAIDS (e.g. aspirin, ibuprofen, diclofenac) inhibit the enzyme cyclooxygenase (COX). There are two isoforms of COX: COX-1, which is produced in most tissues and COX-2, which can be induced by proinflammatory stimuli, tumour promoters and hormones. Both isoforms are blocked by NSAIDS. COX-2 is the rate-limiting step in the synthesis of prostaglandins and therefore NSAIDS inhibit the production of prostaglandins, giving rise to their antiinflammatory, antipyretic and analgesic properties. Inhibition of prostaglandins can also decrease mutagenesis, mitogenesis, angiogenesis and metastasis and increase cellular apoptosis and the antineoplastic activities of $\mathrm{T}$ and B lymphocytes [3].

Oestrogen synthesis is catalysed by the enzyme aromatase cytochrome P450. Experiments in cultured cells have shown that prostaglandin E2 increases aromatase gene expression and thus increases oestrogen synthesis [4]. A recent meta-analysis of results from different trials demonstrated that NSAID use leads to a $22 \%$ reduction in the risk of developing breast cancer [5], but in the light of the knowledge of the above pathways it follows that this reduction should be greatest in women who are ER positive.

\section{Aspirin reduces the risk of ER positive tumours}

The Women's Health Initiative is an ongoing multicentre observational cohort study, designed to address the causes of morbidity and mortality in postmenopausal women. It reported a $21 \%$ reduction in breast cancer risk in women regularly taking NSAIDS. No change in the risk of developing breast cancer was evident in those patients taking acetaminophen (paracetamol, an analgesia with no anti-inflammatory properties) $(n=80,741)$ [1].

In the recent study [2], Terry and colleagues, designed a population based case control study in which they assessed 1508 patients with breast cancer and 1556 control patients. Known breast cancer risk factors were assessed (including parity, breast feeding, family history) along with an assessment of NSAID intake. Patients were categorised as having ever taken aspirin/ibuprofen if they had taken either drug at least once a week for at least a six-month period. As the questionnaire required patients to give information retrospectively there is the possibility of inaccuracies in the recall of NSAID intake. A medical history was detailed for each participant but details on the medical indication for NSAID intake have not been published. Intake of acetaminophen (paracetamol) was also assessed. The ER/PR status was obtained from pathology reports.

Previous use of aspirin was found to be inversely associated with breast cancer risk (OR 0.8; 95\% Cl $0.66-0.97)$. The risk reduction was statistically significant for breast cancer but a trend in reduction was also observed in patients with in situ disease. Both pre- and postmenopausal women showed a reduction in risk, although it was more marked in the postmenopausal group. With respect to hormone receptor status, the risk reduction was seen in all subgroups (ER+ PR+, ER+ 
PR-, ER- PR+) except those patients who were both ER and PR negative. In these patients, NSAID intake bore no correlation to breast cancer risk.

In postmenopausal women taking ibuprofen, a trend towards a decrease in breast cancer risk was seen, however, this was not statistically significant. There was no association evident between breast cancer risk and acetaminophen intake. Dose of NSAID was not assessed but the frequency and duration of use was; patients reporting daily use for up to 5 years showed the greatest risk reduction. As COX is continuously synthesised it follows logically that regular intake of NSAID will be necessary to effectively reduce prostaglandin production with the drawback of an increased risk of gastrotoxicity.

\section{Conclusion}

There is now a wealth of evidence linking aspirin intake to a reduction in breast cancer risk and this study clearly adds the importance of oestrogen receptor status into the debate. Understanding the mechanism of action of aspirin draws emphasis to the importance of COX and prostaglandins in the pathogenesis of breast cancer. Indeed, the enzyme COX-2 is upregulated in human breast cancers [6] and administration of COX-1 and COX-2 inhibitors to mice with established tumours leads to inhibition of tumour growth and a decrease in metastatic capacity [7]. Similarly, administration of NSAIDS to rats with induced mammary carcinogenesis led to a decrease in tumour volume and reduced gene expression of COX-1 and COX-2 [reviewed in 8]. Information regarding aspirin dose is still lacking and several studies have suggested that a high dose $(>100 \mathrm{mg} /$ day) will be necessary [1], which will naturally increase the risk of common adverse effects of NSAIDS, including peptic ulcer disease and gastrointestinal bleeding. Further research into this subject is still necessary; however, aspirin appears to hold an important protective role.

\section{Competing interests}

None declared.

\section{Acknowledgements}

BCRAG and Mr K Horgan for financial support.

\section{References}

1. Harris RE, Chlebowski RT, Jackson RD, Frid DJ, Ascenso JL, Anderson G, Loar A, Rodabough RJ, White E, McTiernan A: Breast cancer and nonsteroidal anti-inflammatory drugs: prospective results from the womn's health initiative. Cancer Res 2003, 63:6096-6101.

2. Terry MB, Gammon MD, Zhang FF, Tawfik H, Teitelbaum SL, Britton JA, Subbaramaiah K, Dannenberg AJ, Neugut Al: Association of frequency and duration of aspirin use and hormone receptor status with breast cancer risk. JAMA 2004, 291: 2433-2440.

3. Gupta RA, Dubois RN: Colorectal cancer prevention and treatment by inhibition of cyclooxygenase-2. Nat Rev Cancer 2001, 1:111-121.

4. Zhao Y, Agarwal VR, MendelsonCR, Simpson ER: Estrogen biosynthesis proximal to a breast tumour is stimulated by
PGE2 via cyclic AMP, leading to activation of promoter II of the CYP19 (aromatase gene). Endocrinology1996, 137:57395742.

5. Khuder SA, Mutgi AB. Breast cancer and NSAID use: a metaanalysis. Br J Cancer 2001, 84:1188-1192.

6. Yoshimura N, Sano $\mathrm{H}$, Okamoto $\mathrm{M}$, Akioks $\mathrm{K}$, Ushigome $\mathrm{H}$, Kadotani Y, Yoahimura R, Nobori S, Higuchi A, Ohmori Y, Nakamura $\mathrm{K}$ : Expression of cyclooxygenase-1 and -2 in human breast cancer. Surg Today 2003, 33:805-811.

7. Kundu N, Fulton AM: Selective cyclooxygenase (COX)-1 or COX-2 inhibitors control metastatic disease in a murine model of breast cancer. Cancer Res 2002, 62:2343-2346.

8. Davies GLS: Cyclooxygenase-2 and chemoprevention of breast cancer. J Steroid Biochem 2003, 86:495-499. 